\title{
Partially coherent stable and spiral beams
}

\author{
T. Alieva, ${ }^{1, *}$ J. A. Rodrigo, ${ }^{1}$ A. Cámara, ${ }^{1}$ and E. Abramochkin ${ }^{2}$ \\ ${ }^{1}$ Universidad Complutense de Madrid, Facultad de Ciencias Físicas, Ciudad Universitaria s/n, Madrid 28040, Spain \\ ${ }^{2}$ Samara branch of P. N. Lebedev Physical Institute of Russian Academy of Sciences, \\ Novo-Sadovaya St., 221, Samara 443011, Russia \\ *Corresponding author: talieva@fis.ucm.es
}

Received June 18, 2013; revised September 2, 2013; accepted September 17, 2013; posted September 18, 2013 (Doc. ID 192358); published October 11, 2013

Stable and spiral coherent beams, which do not change the form of their intensity distribution apart from possible scaling and rotation during propagation and therefore possess self-healing properties, are widely applied in science and technology. On the other hand, it has been found that partially coherent light often provides better output than coherent light. Here we consider two methods for the design and experimental generation of partially coherent stable and spiral beams. (c) 2013 Optical Society of America

OCIS codes: (030.0030) Coherence and statistical optics; (140.3300) Laser beam shaping; (070.2575)

Fractional Fourier transforms; (090.1995) Digital holography.

http://dx.doi.org/10.1364/JOSAA.30.002237

\section{INTRODUCTION}

The generation of optical beams with well-defined spatial structure is required for numerous applications. Often this structure has to be maintained during beam propagation through an optical system. Among the optical systems that are commonly used, we emphasize the homogeneous media and rotationally invariant systems containing spherical lenses and mirrors or quadratic refractive index fibers. Beams that do not change the form of their intensity distribution, except of the possible scaling and rotation during propagation through such systems, are referred to as stable and spiral beams, respectively. The design of a coherent monochromatic stable beam is based on the search for the eigenfunctions of the operator describing the system. In the case of spiral beams, the rotator operator is added to the system operator [1]].

In paraxial approximation, the evolution of the complex field amplitude of a coherent monochromatic beam, $f(\mathbf{r}, \alpha)$, in a rotationally symmetric system can be described, apart from the scaling and quadratic phase, by the symmetric fractional Fourier transform (sFrFT) [2- $\underline{5}]$

$$
\begin{aligned}
f(\mathbf{r}, \alpha)= & \frac{1}{\mathrm{i} s^{2} \sin \alpha} \\
& \times \int \exp \left[\mathrm{i} \pi \frac{\left(r^{2}+r_{i}^{2}\right) \cos \alpha-2 \mathbf{r}_{i} \mathbf{r}^{t}}{s^{2} \sin \alpha}\right] f\left(\mathbf{r}_{i}, 0\right) \mathrm{d} \mathbf{r}_{i} .
\end{aligned}
$$

Here $\mathbf{r}=(x, y)$ is a two-dimensional position vector at any plane transverse to the beam propagation direction, the subindex $i$ indicates the input plane, $s$ is a system normalization parameter, and $\alpha$ is an angle defining the rotation in the position-spatial frequency space. Thus, the propagation of the intensity distribution through an optical fiber with a quadratic profile of refractive index $n(r)^{2}=n_{0}^{2}\left(1-n_{1}^{2} r^{2}\right)$ is described by the $\operatorname{sFrFT}\left|f_{\text {fiber }}(\mathbf{r}, z)\right|^{2}=|f(\mathbf{r}, \alpha)|^{2}$ with $\alpha=$ $n_{1} z$ and scaling $s=\sqrt{\lambda / n_{1}}$, where $\lambda$ is the wavelength in the medium with refractive index $n_{0}$. For the case of the propagation in a homogeneous medium of refractive index $n_{0}$ at distance $z$ (Fresnel diffraction) $\left|f_{\text {Fresnel }}(\mathbf{r}, \boldsymbol{z})\right|^{2}=$ $|\cos \alpha f(\cos \alpha \mathbf{r}, \alpha)|^{2}$, where $\alpha=\arctan \left(\lambda z / s^{2}\right)$ [3]. It is easy to see that in the last case, the angle $\alpha$ belongs to the interval $[0, \pi / 2)$, where the value $\pi / 2$ corresponds to the Fraunhofer diffraction approximation.

A similar evolution of the intensity distribution in rotationally symmetric first-order optical systems [4, apply the eigenfunctions to the sFrFT system in the design of structurally stable beams. Alternatively, the eigenfunctions for the Fresnel operator that describes in paraxial approximation the beam propagation through homogeneous media can be used. The eigenfunctions for both operators are well known. Thus, Hermite-Gaussian (HG) [2,6,7], LaguerreGaussian (LG), or more sophisticated Hermite-LaguerreGaussian (HLG) [8-10] and Ince-Gaussian (IG) [11-13] modes are eigenfunctions for the sFrFT system. Moreover, all these families form complete orthogonal sets and can be used for the construction of other beams [14] with intensity invariance under propagation through a quadratic refractive index medium with an appropriate scaling parameter $s$. Nevertheless, during the propagation in a homogeneous medium, these beams maintain the form of the intensity distribution but not the scale. In contrast, the intensity distribution of the eigenfunctions for the Fresnel operator, for example, the Bessel modes, is invariant during propagation through the homogeneous media, but its scale changes in other rotationally symmetric systems. Since the eigenfunctions of the Fresnel operator possess infinite energy that is not physically realizable, they have to be apodized by a Gaussian function, which yields the Bessel-Gaussian beams [15]. Such beams slightly change the form of their intensity distribution during propagation through the considered systems. Therefore, here we use the sets of the sFrFT eigenfunctions as the base for the structurally stable beam design.

Coherent stable beams (cSTBs), such as HG, LG, HLG, and IG modes, and coherent spiral beams (cSPBs) [16-20] are 
widely used nowadays for particle manipulation [21,22], resolution improvement in microscopy [23-26], free-space communication [27], atmosphere monitoring [28,29], etc. Moreover, it has been shown that as well as the Bessel beams, the cSTBs (and similarly, the cSPBs) are able to reconstruct their forms after propagation through small obstacles. This property also referred to as self-healing makes them more attractive for the mentioned applications. On the other side, it has been found that partially coherent light often provides better output than coherent light. In particular, partially coherent light has beneficial applications in imaging for speckle reduction [30], beam shaping in lithography [31], inertial plasma confinement, and free-space communication [32-35]. The combination of both properties-beam stability and partial coherence-promises additional advantages.

While a monochromatic paraxial coherent beam is described by a complex field amplitude at the $x y$ plane transverse to the propagation direction, which depends on only two variables, the partially coherent beam is characterized by a four-dimensional function known as mutual intensity (MI), which is defined as $\Gamma\left(\mathbf{r}_{1}, \mathbf{r}_{2}\right)=\left\langle f\left(\mathbf{r}_{1}\right) f^{*}\left(\mathbf{r}_{2}\right)\right\rangle$. Here, the * means complex conjugation and $\langle\cdot\rangle$ stands for ensemble averaging, which is omitted in the coherent case. The MI describes the field correlation at two points at the same time moment. The equal-time degree of coherence, also called the complex coherence factor (see [36]) defined as $\gamma\left(\mathbf{r}_{1}, \mathbf{r}_{2}\right)=\Gamma\left(\mathbf{r}_{1}, \mathbf{r}_{2}\right) / \sqrt{\Gamma\left(\mathbf{r}_{1}, \mathbf{r}_{1}\right) \Gamma\left(\mathbf{r}_{2}, \mathbf{r}_{2}\right)}$, or its modulus are usually used for the analysis of the spatial coherence. Further, $\gamma\left(\mathbf{r}_{1}, \mathbf{r}_{2}\right)$ will be briefly referred to as a degree of coherence. The partially coherent beam, whose intensity distribution $\Gamma(\mathbf{r}, \mathbf{r})$ does not change apart from the scaling and rotation during its propagation through homogeneous media, will be referred to as a partially coherent stable beam (pSTB) and a partially coherent spiral beam (pSPB), correspondingly.

For the generation of the coherent stable and spiral beams, computer-generated holograms (CGHs) are used. The complex field amplitude is encoded in the CGH that is implemented into the spatial light modulator (SLM). The coherent reference beam (often approximated by a plane wave) is used for hologram reading.

The synthesis of partially coherent beams is based on two main approaches [37]. In the first one, Schell-model beams with determined intensity distribution and homogeneous amplitude of the coherence degree $\left|\gamma\left(\left|\mathbf{r}_{1}-\mathbf{r}_{2}\right|\right)\right|$ usually expressed by a Gaussian function are generated. The propagation of partially coherent HG and LG beams constructed in this way, which is the particular case of the pSTBs, has been theoretically studied in $[38,39]$. Another approach considered, for example, in [40-42], uses the coherent mode expansion of the MI [43].

In this paper, we consider both methods of designing and generating pSTBs and pSPBs by digital holography. The experimental verification of the proposed techniques is performed using the optical setup that allows an automated implementation of the sFrFT for the running parameter $\alpha$ that is described in [44]. The setup consists of two SLMs and a digital camera located at fixed positions. Parameter $\alpha$ is varied by changing the lens power implemented by the SLMs.

The paper is organized as follows. In Section $\underline{2}$, we briefly review the design of the cSTBs and the cSPBs. Section 3 describes and experimentally verifies the method of the pSTB and pSPB generation using a partially coherent reference beam to read the hologram which encodes the associated coherent beam. Another method of the pSTB and pSPB design that is based on the MI mode expansion is considered in Section 4. The self-reconstructing properties of the pSTBs and $\mathrm{pSPB}$ are discussed in Section $\underline{5}$. Finally, Section $\underline{6}$ presents the conclusions.

\section{DESIGN OF COHERENT STABLE AND SPIRAL BEAMS}

For our beam design, it is preferable, but not essential, to use a complete orthonormal basis of modes with rotationally invariant amplitude and an azimuthally dependent phase $f(r, \theta)=a(r) \exp (i l \theta)$, such as the LG modes:

$$
\begin{aligned}
\mathrm{LG}_{m, n}(\mathbf{r}, w)= & {\left[\frac{2(\min \{m, n\}) !}{(\max \{m, n\}) !}\right]^{1 / 2} \exp \left(\frac{-\pi r^{2}}{w^{2}}\right) } \\
& \times\left(\frac{\sqrt{2 \pi} r}{w}\right)^{|m-n|} \exp [i(m-n) \theta] L_{\min \{m, n\}}^{(|m-n|)}\left(\frac{2 \pi r^{2}}{w^{2}}\right) .
\end{aligned}
$$

Here $L_{p}^{(l)}(\cdot)$ denotes the generalized Laguerre polynomials, and the two-dimensional vector $\mathbf{r}=(x, y)=(r \cos \theta, r \sin \theta)$ represents the spatial coordinates. We emphasize that the LG mode $\operatorname{LG}_{m, n}(\mathbf{r}, w)$ for $w=s$ is an eigenfunction for the symmetric fractional FT [see Eq. (1)] whose eigenvalue $\exp [-\mathrm{i}(m+n+1) \alpha]$ depends on the parameter of transformation, the angle $\alpha$, and the indices of the mode. The eigenvalue determines the Gouy phase acquired by the mode during the propagation through the homogeneous medium.

It is easy to see that the coherent linear superposition of the LG modes with a constant sum of indices $m+n=N=$ const forms another stable beam. In particular, the HG, HLG, and IG beams, among many others, can be written in this form.

The LG mode $\mathrm{LG}_{m, n}(\mathbf{r}, w)$ is also an eigenfunction for the operator associated with the rotation at the $x y$ plane. The eigenvalue $\exp [\mathrm{i}(m-n) \beta]$ corresponds to the counterclockwise rotation at angle $\beta$. Because the LG modes are eigenfunctions for the symmetric fractional FT and the image rotator operator, they are suitable for the construction of the cSPBs. These beams preserve their intensity distribution, apart from a rotation during the propagation in a certain quadratic refractive index medium and apart from the rotation and scaling during the propagation in other rotationally symmetric firstorder systems including the homogeneous media. Indeed, it has been shown in $[\underline{1}, \underline{20}]$ that the linear combination of the LG modes with arbitrary complex numbers $c_{m, n}$,

$$
\Psi(\mathbf{r}, w, v)=\sum_{m, n} c_{m, n} \mathrm{LG}_{m, n}(\mathbf{r}, w),
$$

whose indices $m$ and $n$ satisfy the relation

$$
m(1-v)+n(1+v)=\text { const, }
$$

forms the cSPB. For irrational $v$, Eq. (4) holds only for one LG mode. The absolute value of the rational parameter $v$ defines the velocity of the intensity rotation (number of loops) during the beam propagation through the $\mathrm{sFrFT}$ system in the angle interval of $2 \pi$, while the sign of $v$ indicates the rotation direction. For example, for $v=1$, the following family of spiral beams, 


$$
\Psi_{n}(\mathbf{r}, w, 1)=\sum_{m=0}^{\infty} c_{m, n} \mathrm{LG}_{m, n}(\mathbf{r}, w)
$$

with fixed $n$ is obtained. During the propagation of such beam through a homogeneous medium, the intensity distribution rotates at $\pi / 2$. If the designed cSPB is an eigenfunction for the sFrFT for angle $\alpha=2 \pi / k$ with an integer $k$, then $v=k$. The rotation velocity can be less than 1 if the cSPB is an eigenfunction for the rotator operator at angle $\beta=2 \pi / l$, where $l$ is an integer. Thus, the cSPB characterized by $v=k / l$ is autoreciprocal under the rotation at the $x y$ plane at the angle $\beta=2 \pi / l$ and is an eigenfunction for the sFrFT for angle $\alpha=2 \pi / k$. In general, any pair of modes $\mathrm{LG}_{m_{0}, n_{0}}(\mathbf{r}, w)$ and $\mathrm{LG}_{m, n}(\mathbf{r}, w)$ with nonequal eigenvalues for the rotator $(m-n) \neq\left(m_{0}-n_{0}\right)$ and the fractional FT $(m+n) \neq\left(m_{0}+n_{0}\right)$ operators forms a spiral beam whose rotation velocity is defined by

$$
v=\frac{(m+n)-\left(m_{0}+n_{0}\right)}{(m-n)-\left(m_{0}-n_{0}\right)} .
$$

For the design of a cSPB that follows a determined curve, another technique discussed in [18] is more effective. While the resulting beam can be presented also as a superposition of the LG modes (3) following the rule (4), the technique does not require the determination of appropriate expansion coefficients.

\section{PARTIALLY COHERENT STABLE AND SPIRAL BEAMS GENERATED BY A SINGLE HOLOGRAM}

Let us first consider the method for generating a partially coherent beam whose intensity distribution resembles the form of a stable or spiral coherent beam. The signal $f_{C}(\mathbf{r})$ is encoded into a phase-only hologram following the approach reported in [45]. This hologram is addressed into the SLM (Holoeye, Pluto, pixel size of $8 \mu \mathrm{m}$ ) illuminated with a homogeneously randomized laser beam obtained using a rotating ground glass diffuser. Indeed, a laser beam approximated by a Gaussian function and passing through the diffuser is transformed into a spatially incoherent Gaussian beam. According to the van Cittert-Zernike theorem [36], the degree of coherence of the resulting beam changes during the propagation through an optical system. Suppose that at the SLM plane the MI of the reference beam $\Gamma_{R}\left(\mathbf{r}_{1}, \mathbf{r}_{2}\right)$ is described by

$$
\Gamma_{R}\left(\mathbf{r}_{1}, \mathbf{r}_{2}\right)=I_{0} \exp \left[-\pi\left|\mathbf{r}_{1}-\mathbf{r}_{2}\right|^{2} / w_{0}^{2}\right]
$$

The light modulation by the SLM treated in the thin screen approximation yields after the appropriate filtering of the desired diffraction order the partially coherent beam described by the MI,

$$
\Gamma\left(\mathbf{r}_{1}, \mathbf{r}_{2}\right)=f_{C}\left(\mathbf{r}_{1}\right) f_{C}^{*}\left(\mathbf{r}_{2}\right) \Gamma_{R}\left(\mathbf{r}_{1}, \mathbf{r}_{2}\right)=\Gamma_{R}\left(\mathbf{r}_{1}, \mathbf{r}_{2}\right) \Gamma_{C}\left(\mathbf{r}_{1}, \mathbf{r}_{2}\right),
$$

where $\Gamma_{C}$ is the MI of the coherent beam $f_{C}(\mathbf{r})$. Then, if $f_{C}(\mathbf{r})$ corresponds to a cSTB (or cSPB), then the resulting beam for a certain propagation distance can be considered as a pSTB (or pSPB).

As an example, let us experimentally study the propagation of the pSPB associated with the cSPB described as the coherent composition of LG modes [see Eq. (ㅁ)]:

$$
\Psi_{2}(\mathbf{r}, w, 1)=\sum_{n=1}^{8} \frac{\sqrt{(3 n+2) !}}{(3 n) !} 3^{3 n} \operatorname{LG}_{3 n+2,2}(\mathbf{r}, w),
$$

with $w=0.73 \mathrm{~mm}$. The MI of the reference beam has a Gaussian form with $w_{0}=0.45 \mathrm{~mm}$. The evolution of the intensity distributions of both beams propagated through the sFrFT system is displayed in Fig. 1 (see Media 1 online). The intensity patterns presented in all of the figures over the work have the size $2 \mathrm{~mm} \times 2 \mathrm{~mm}$. We recall that this intensity evolution coincides, apart from the scaling, with the evolution during the propagation through a homogeneous medium. The synchronous rotation of the intensity distribution of the beams during the propagation is observed. Moreover, the use of the partially coherent beam allows us to reduce significantly the undesirable speckle effect, maintaining the spatial structure of the intensity of the coherent beam.

Nevertheless, the intensity distribution of the pSPB (or the pSTB) obtained this way preserves its form only for a relatively high coherence degree. In general, contrary to the coherent case, the amplitude of the MI of the partially coherent beam changes during the propagation, even for the Gaussian partially coherent reference beam. This leads to the intensity changes clearly observed in Fig. 2 , where the evolution of the cSTB, $\operatorname{LG}_{5,4}(\mathbf{r}, w)$, Fig. 2(a), and the associated pSTBs for different coherence parameters $w_{0}=0.45 \mathrm{~mm}$, Fig. 2(b), and $w_{0}=0.16 \mathrm{~mm}$, Fig. $2(\mathrm{c})$ are shown. We observe that at the plane $\alpha=0$, the intensity distributions of the beams with different $w_{0}$ are very similar, while they have different evolution during the beam propagation. For the almost incoherent beam corresponding to $w_{0}=0.16 \mathrm{~mm}$, the intensity structure is washed out already for a small propagation distance [see Fig. 2(c)]. However, the more coherent beam preserves the intensity distribution structure even at the Fourier conjugated plane [see Fig. 2(b)].

Therefore, the distance where the intensity distribution of the partially coherent beam generated by this method has a stable structure, highly depends on the coherence radius, the beam width, and the beam complexity. Theoretical consideration of the evolution of the MI of the particular pSTBspartially coherent HG beams-during their propagation has been considered in [38]. (a)

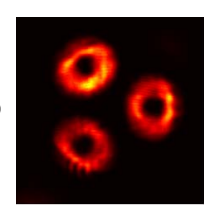

(b)

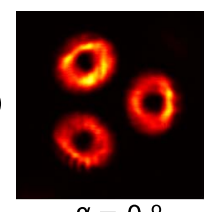

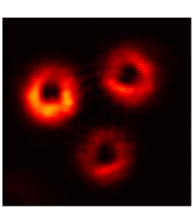

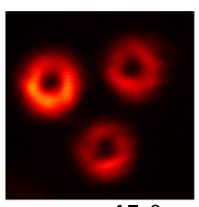

$\alpha=45$ 음
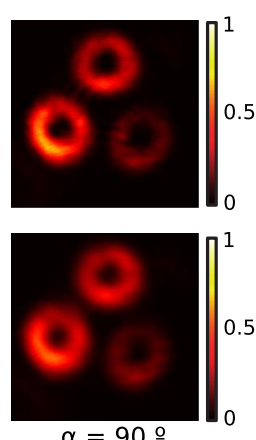

Fig. 1. (a) Evolution of the intensity distribution of the cSPB given by Eq. (9) and (b) the associated pSPB during propagation through the $\mathrm{sFrF} \overline{\mathrm{T}}$ system. See Media 1 online. 
(a)

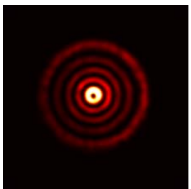

(b)

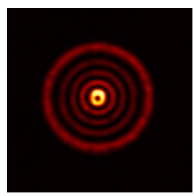

(c)

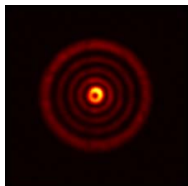

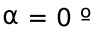
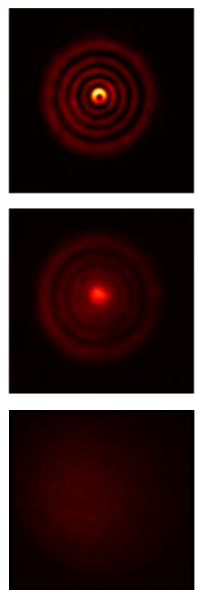

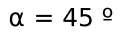
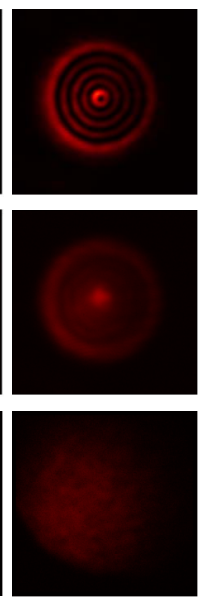

$\alpha=90 \stackrel{0}{\circ}$

Fig. 2. (a) Evolution of the intensity distribution of the cSTB, $\mathrm{LG}_{5,4}(\mathbf{r}, w)$, and (b), (c) the associated pSPB of different degrees of coherence during propagation through the sFrFT system. The radius of the coherence degree of the beam shown in (b) is larger than in (c). See Media 2 online.

\section{PARTIALLY COHERENT STABLE AND SPIRAL BEAMS GENERATED BY HOLOGRAM MULTIPLEXING}

Another way to generate the pSTBs or pSPBs is based on the MI mode expansion. It is known [43] that any MI can be expressed as

$$
\Gamma\left(\mathbf{r}_{1}, \mathbf{r}_{2}\right)=\sum_{n} a_{n} \psi_{n}\left(\mathbf{r}_{1}\right) \psi_{n}^{*}\left(\mathbf{r}_{2}\right),
$$

where the set $\left\{\psi_{n}(\mathbf{r})\right\}$ forms an orthogonal basis and $a_{n}$ are real non-negative constants. In particular, a partially coherent Gaussian Schell-model beam can be decomposed in HG, LG, or HLG modes depending on its orbital angular momentum [46]. Partially coherent vortex beams with a separable helical phase have been synthesized by this method [41,42] using the LG modes with the same topological charge as functions $\psi_{n}(\mathbf{r})$.

Spatial or temporal hologram multiplexing can be used for the synthesis of partially coherent light composed by a few orthogonal modes [37]. In the case of spatial multiplexing, a laser with a low longitudinal length of coherence $L$ (about $1 \mathrm{~cm}$ ) is divided by beam splitters into $N$ beams, where $N$ is the number of modes used in the MI decomposition. Each of these $N$ beams is modulated by a hologram that encodes the mode $\psi_{n}(\mathbf{r})$ (here, $\left.n=1,2, \ldots, N\right)$ implemented by its corresponding SLM. The modulated beams are combined incoherently. Therefore, the effective optical paths of the beams have to differ from one another more than the distance $L$. A similar scheme has been used in [32,42]. In particular, in [32], the modes from the decomposition have been used as independent channels in order to increase of the information transmission capacity in free-space communication.

Alternatively, time hologram multiplexing can be used for partially coherent beam generation. In this case, the holograms encoding the modes are implemented consecutively using the same SLM. However, the temporal response of the system, which makes use of partial coherence, has to be larger than the time of exposition of all $N$ holograms.

For the generation of the pSTBs, any basis of orthogonal stable beams, such as the LG, HG, IG, HLG, among many

others, can be used. The incoherent sum of the modes belonging to the same basis generates the pSTB whose MI does not change during the propagation in the homogeneous media, apart from the scaling and quadratic phase. The complex structure of the field coherence can be obtained by applying this method.

As an example, we consider the pSTB composed of two LG modes $\psi_{1}(\mathbf{r})=\mathrm{LG}_{n, m}(\mathbf{r}, w)$ and $\psi_{2}(\mathbf{r})=\mathrm{LG}_{m, n}(\mathbf{r}, w)$ with $a_{1}=a_{2}=1 / 2$ and $m \neq n$. The intensity distribution of this beam coincides with the intensity distribution of the cSTB $\mathrm{LG}_{n, m}(\mathbf{r}, w)$ and $\mathrm{LG}_{m, n}(\mathbf{r}, w)$, while its coherence degree written in polar coordinates is expressed by

$$
\gamma\left(r_{1}, \theta_{1} ; r_{2}, \theta_{2}\right)=\cos \left[(m-n)\left(\theta_{1}-\theta_{2}\right)\right] .
$$

Thus, the field at two points with the same angular coordinate $\theta$ and azimuthal coordinates $\left(\theta_{1}-\theta_{2}\right) /(m-n)=\pi$ is completely correlated, $|\gamma|=1$. However, the field at the points, such that

$$
\left(\theta_{1}-\theta_{2}\right) /(m-n)=\pi / 2+\pi k,
$$

where $k$ is an integer, is uncorrelated, $|\gamma|=0$.

The intensity distribution of the beam constructed as a incoherent sum of the LG modes $\psi_{1}(\mathbf{r})=\mathrm{LG}_{0,0}(\mathbf{r}, w)$, $\psi_{2}(\mathbf{r})=\mathrm{LG}_{0,1}(\mathbf{r}, w)$, and $\psi_{3}(\mathbf{r})=\mathrm{LG}_{1,2}(\mathbf{r}, w)$ with weights $a_{1}=1.25, a_{2}=4.4$, and $a_{3}=1$ is displayed in Fig. 3(a). Even though the intensity distribution of this beam is almost homogeneous, the modulus of its coherence degree $\left|\gamma\left(\mathbf{r}_{0}, \mathbf{r}\right)\right|$ is not, as can be seen in Fig. 3(b), where the radial profiles of

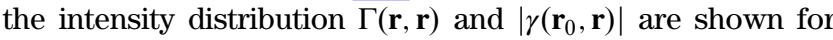
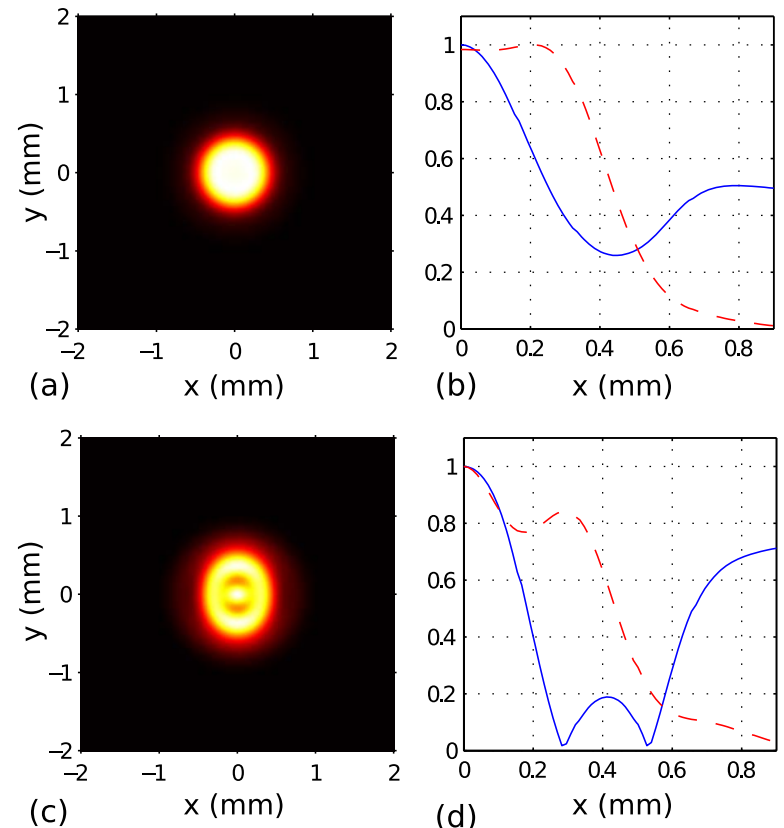

Fig. 3. (a) Intensity distribution of the pSTB constructed as an incoherent sum of the orthogonal modes $\psi_{1}(\mathbf{r})=\mathrm{LG}_{0,0}(\mathbf{r}, w)$, $\psi_{2}(\mathbf{r})=\mathrm{LG}_{0,1}(\mathbf{r}, w)$, and $\psi_{3}(\mathbf{r})=\mathrm{LG}_{1,2}(\mathbf{r}, w)$ with weights $a_{1}=1.25$, $a_{2}=4.4$, and $a_{3}=1$. (b) Simulated profiles of $\Gamma(\mathbf{r}, \mathbf{r})$ (dashed line) and $\left|\gamma\left(\mathbf{r}_{0}, \mathbf{r}\right)\right|$ (continuous line) for $\mathbf{r}_{0}=(0,0)^{t}$ and $\mathbf{r}=(x, 0)^{t}$ of this beam. (c) Intensity distribution of the pSTB constructed as an incoherent sum of the orthogonal modes $\psi_{1}(\mathbf{r})=\mathrm{LG}_{2,0}(\mathbf{r}, w)+$ $\mathrm{LG}_{1,1}(\mathbf{r}, w), \psi_{2}(\mathbf{r})=\mathrm{LG}_{1,2}(\mathbf{r}, w)$, and $\psi_{3}(\mathbf{r})=\mathrm{LG}_{1,0}(\mathbf{r}, w)$ with weights $a_{1}=1, a_{2}=3.5$, and $a_{3}=2$. (d) Simulated profiles of $\Gamma(\mathbf{r}, \mathbf{r})$ (dashed line) and $\left|\gamma\left(\mathbf{r}_{0}, \mathbf{r}\right)\right|$ (continuous line) for $\mathbf{r}_{0}=(0,0)^{t}$ and $\mathbf{r}=(x, 0)^{t}$ of this beam. 
$\mathbf{r}_{0}=(0,0)$ and $\mathbf{r}=(x, 0)$. In Figs. 3(b), 3(d), and $\underline{4(\mathrm{c})}$, the intensity profiles are given in normalized units. Note that the partially coherent vortex beams with a separable helical phase $[\underline{41}, \underline{42}]$ have been constructed in this way, and therefore, they also belong to the class of pSTBs.

It is easy to see that the partially coherent beams constructed using the HG basis have axial symmetry, while the ones generated from the LG basis are central symmetric. As we have mentioned in Section 2, there are other stable beams that can be written as a linear composition of the LG modes with a constant index sum $N=m+n$. The incoherent composition of such orthogonal beams, which means that they contain disjoint LG mode subsets, also corresponds to a stable partially coherent beam. In order to find the general form for the partially coherent stable beams, let us decompose its MI in the double set of LG modes:

$$
\Gamma\left(\mathbf{r}_{1}, \mathbf{r}_{2}\right)=\sum_{m, n} \sum_{m^{\prime}, n^{\prime}}\left\langle c_{m, n} c_{m^{\prime}, n^{\prime}}^{*}\right\rangle \mathrm{LG}_{m, n}\left(\mathbf{r}_{1}, w\right) \mathrm{LG}_{m^{\prime}, n^{\prime}}^{*}\left(\mathbf{r}_{2}, w\right),
$$

where $\left\langle c_{m, n} c_{m^{\prime}, n^{\prime}}^{*}\right\rangle$ are the correlation coefficients of the expansion. Analogously, the HG (see, for example, [47,48]) or HLG set can be used. Note that in contrast to Eq. (10), the LG modes in this expansion are not necessarily eigenmodes of the MI. The propagation of the beam with an appropriate parameter $w=s$ through the sFrFT system leads to the following change of the MI:

$$
\begin{aligned}
\Gamma\left(\mathbf{r}_{1}, \mathbf{r}_{2}, \alpha\right)= & \sum_{m, n} \sum_{m^{\prime}, n^{\prime}}\left\langle c_{m, n} c_{m^{\prime}, n^{\prime}}^{*}\right\rangle \\
& \times \exp \left[-i\left(m+n-m^{\prime}-n^{\prime}\right) \alpha\right] \\
& \times \mathrm{LG}_{m, n}\left(\mathbf{r}_{1}, w\right) \mathrm{LG}_{m^{\prime}, n^{\prime}}^{*}\left(\mathbf{r}_{2}, w\right)
\end{aligned}
$$

Analyzing this equation, we conclude that only the modes with the same index sum $m+n=m^{\prime}+n^{\prime}=N_{k}$ in the MI mode decomposition can be correlated between each other, forming by this way a new coherent stable mode $\psi_{k}$. All other correlation coefficients vanish $\left\langle c_{m, n} c_{m^{\prime}, n^{\prime}}^{*}\right\rangle=0$ if $m \neq m^{\prime}$ and $n \neq n^{\prime}$. As an example, we display in Fig. 3(c) the intensity distribution of the pSTB generated as an incoherent sum of the following orthogonal modes: $\psi_{1}(\mathbf{r})=\mathrm{LG}_{2,0}(\mathbf{r}, w)+\mathrm{LG}_{1,1}(\mathbf{r}, w)$, $\psi_{2}(\mathbf{r})=\mathrm{LG}_{1,2}(\mathbf{r}, w)$, and $\psi_{3}(\mathbf{r})=\mathrm{LG}_{1,0}(\mathbf{r}, w)$, with the coefficients $a_{1}=1, a_{2}=3.5$, and $a_{3}=2$. In Fig. $3(\mathrm{~d})$, the radial profiles of the intensity distribution $\Gamma(\mathbf{r}, \mathbf{r})$ and $\left.\overline{\mid \gamma\left(\mathbf{r}_{0}\right.}, \mathbf{r}\right) \mid$ are shown for $\mathbf{r}_{0}=(0,0)$ and $\mathbf{r}=(x, 0)$. We observe that the beam is stable even though it has a rather complex coherence structure. Note that the use of beams with variable coherence is beneficial for the development of imaging techniques [49].

The pSPBs can be generated in a similar fashion. In this case, the orthogonal cSPBs, $\operatorname{SP}_{n}(\mathbf{r}, w, v)$, with equal rotation velocity $v$ and the stable modes, $\mathrm{ST}_{m}(\mathbf{r}, w)$, with rotationally symmetric intensity distribution are composed incoherently. The resulting MI then can be expressed as

$$
\begin{aligned}
\Gamma\left(\mathbf{r}_{1}, \mathbf{r}_{2}\right)= & \sum_{n} a_{n} \mathrm{SP}_{n}\left(\mathbf{r}_{1}, w, v\right) \mathrm{SP}_{n}^{*}\left(\mathbf{r}_{2}, w, v\right) \\
& +\sum_{m} b_{m} \mathrm{ST}_{m}\left(\mathbf{r}_{1}, w\right) \mathrm{ST}_{m}^{*}\left(\mathbf{r}_{2}, w\right),
\end{aligned}
$$

where $a_{n}$ and $b_{m}$ are nonnegative constants. We emphasize that all modes in this decomposition are orthogonal. The constant $v$ defines the rotation velocity of the intensity

distribution of such pSPB during the beam propagation through the sFrFT system.

The evolution during propagation through the sFrFT system of the intensity distribution of the partially coherent beam experimentally generated by the time multiplexing of two holograms encoding the $\mathrm{cSPB}\left[\psi_{1}(\mathbf{r})=\Psi_{2}(\mathbf{r}, w, 1)\right.$, see Eq. (9)] and the cSTB $\psi_{2}(\mathbf{r})=\mathrm{LG}_{5,4}(\mathbf{r}, s)$ with equal weights is shown in Fig. 4(a) (see Media 3 online). We observe the rotation of the intensity distribution with the velocity defined by $v=1$. The stable partially coherent beam and the spiral one have a rather complex coherence structure. The intensity distribution of this beam, Fig. 4(b), and the radial profiles of the intensity distribution, $\Gamma(\mathbf{r}, \mathbf{r})$, and the $\left|\gamma\left(\mathbf{r}_{0}, \mathbf{r}\right)\right|$ for $\mathbf{r}_{0}=\left(x_{0}, 0\right)$ and $\mathbf{r}=(x, 0)$ for $x_{0}=0.1 \mathrm{~mm}$ calculated numerically are displayed in Fig. $4(\mathrm{c})$. There are points $x$ with nonzero intensities where the field is uncorrelated with one at point $x_{0}$.

\section{SELF-HEALING OF THE PARTIALLY COHERENT STABLE AND SPIRAL BEAMS}

The propagation of the beam possessing structural stability through inhomogeneous media or small obstacles has recently attracted the attention of the scientific community. The reconstruction of the beam form after such distortions is desirable for different applications, including, for example,

(a)

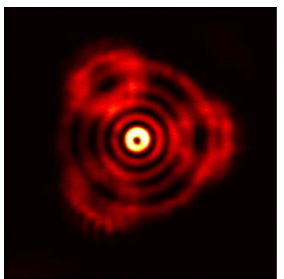

$\alpha=0$ o

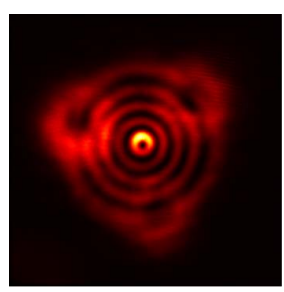

$\alpha=44 \stackrel{\circ}{2}$

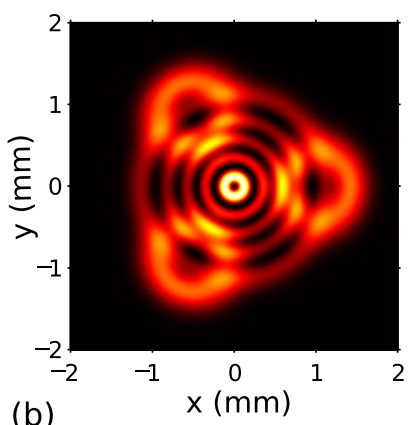

(b)

Fig. 4. (a) Evolution of the intensity distribution of the pSPB constructed as an incoherent sum with equal weights of two orthogonal beams, the cSPB $\Psi_{2}(\mathbf{r}, w, 1)$, see Eq. (9), and the $\operatorname{cSTB~LG}_{5,4}(\mathbf{r}, w)$ during propagation through the sFrFT system. Media 3 online. (b) Simulated intensity distribution of this beam. (c) Simulated profiles of $\Gamma(\mathbf{r}, \mathbf{r})$ (dashed line) and the $\left|\gamma\left(\mathbf{r}_{0}, \mathbf{r}\right)\right|$ (continuous line) for $\mathbf{r}_{0}=\left(x_{0}, 0\right)$ where $x_{0}=0.1 \mathrm{~mm}$ and $\mathbf{r}=(x, 0)$ of this beam. 
(a) cSPB

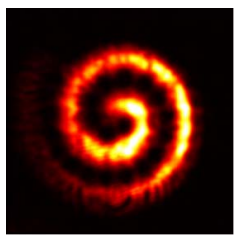

(b) pSPB

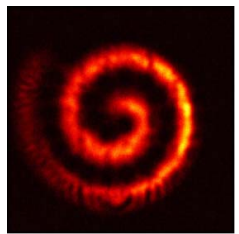

(c) cSPB

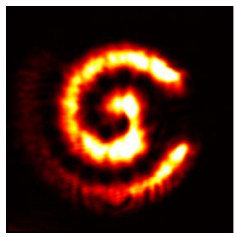

(d)

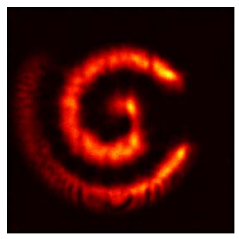

$\alpha=0$ o
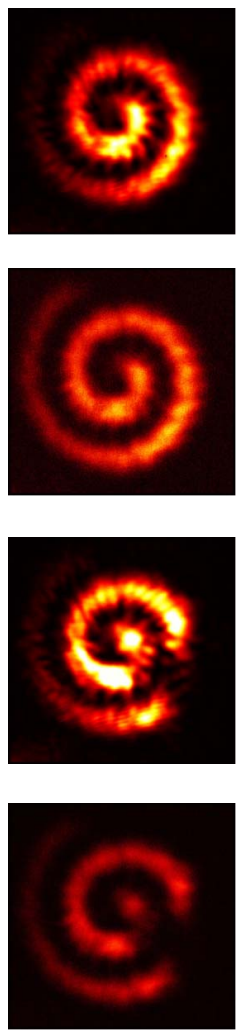

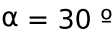
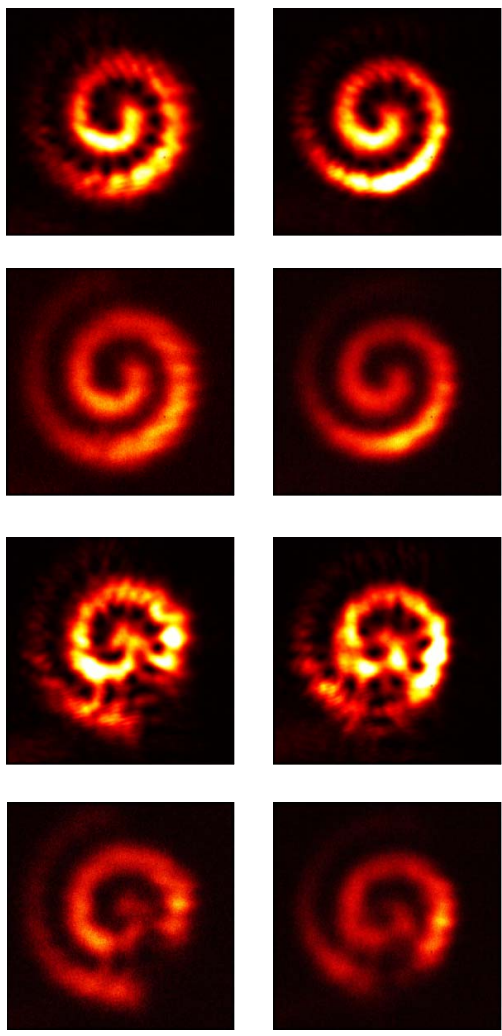

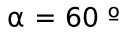

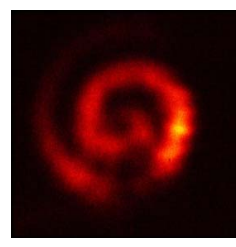

$\alpha=90$ 은

Fig. 5. (a) Evolution of the intensity distribution of the cSPB and (b) the associated pSPB during the propagation through the sFrFT system. (c) Evolution of the intensity distribution of the cSPB and (d) the associated pSPB disturbed by an obstacle at $\alpha=0$ during the propagation through the sFrFT system.

optical trapping [50] or optical free-space communication. This property can be explained easily by the filtering theory [51]. Here we experimentally demonstrate that the pSTBs and pSPBs are also self-healing. Since a quantitative criterion for the estimation of the beam degradation after the healing has not been proposed yet, here we restrict ourselves to the experimental comparison of the self-reconstruction of the spiral coherent and partially coherent beams shown in Fig. 5 . The cSPB corresponding to the Archimedes spiral is synthesized following the method proposed in [18]. Partially coherent beams are generated by applying the single hologram technique discussed in Section 3 for the coherence parameter $w_{0}=0.45 \mathrm{~mm}$. We observe that both types of beams roughly recover their forms after being disturbed by an opaque obstacle whose shape is clearly seen in the intensity patterns corresponding to $\alpha=0$. It seems that the partially coherent beam better recovers its form than the coherent one. It can be explained by a more pronounced interference effect caused by the distortions in the coherent beam that prevents the beam from reconstructing.

The pSTBs and the pSPBs generated by the second method considered in Section $\underline{4}$ also demonstrate the selfreconstruction behavior because they are composed by independent modes, all of which possess this property.

\section{CONCLUSIONS}

We have shown that the pSTBs and pSPBs have properties similar to the ones of their coherent counterparts. In addition, they offer some benefits corresponding to partially coherent light, such as speckle reduction and an increase of the information encoding capacity. The complex structure of the degree of coherence, which can be encoded into the beams synthesized by the second method, is attractive for the development of new imaging techniques and protocols for information transmission in optical communication. The use of digital holography for the generation of these beams opens a way for their wide application.

\section{ACKNOWLEDGMENTS}

The Spanish Ministerio de Economía y Competitividad is acknowledged for the financial support of the project through Grant No. TEC2011-23629.

\section{REFERENCES}

1. T. Alieva, E. Abramochkin, A. Asenjo-Garcia, and E. Razueva, "Rotating beams in isotropic optical system," Opt. Express 18, 3568-3573 (2010).

2. H. M. Ozaktas, Z. Zalevsky, and M. A. Kutay, The Fractional Fourier Transform with Applications in Optics and Signal Processing (Wiley, 2001).

3. T. Alieva, V. Lopez, F. A. Lopez, and L. B. Almeida, "The fractional Fourier transform in optical propagation problems," J. Mod. Opt. 41, 1037-1044 (1994).

4. R. Simon and N. Mukunda, "Iwasawa decomposition in firstorder optics: universal treatment of shape-invariant propagation for coherent and partially coherent beams," J. Opt. Soc. Am. A 15, 2146-2155 (1998).

5. T. Alieva and M. J. Bastiaans, "Properties of the linear canonical integral transformation,” J. Opt. Soc. Am. A 24, 3658-3665 (2007). 
6. D. Mendlovic and H. M. Ozaktas, "Fractional Fourier transforms and their optical implementation: I,” J. Opt. Soc. Am. A 10, 1875-1881 (1993).

7. H. M. Ozaktas and D. Mendlovic, "Fractional Fourier transforms and their optical implementation: II,” J. Opt. Soc. Am. A 10, 2522-2531 (1993)

8. E. Abramochkin and V. Volostnikov, "Beam transformations and nontransformed beams," Opt. Commun. 83, 123-135 (1991).

9. E. G. Abramochkin and V. G. Volostnikov, "Generalized Gaussian beams,” J. Opt. A 6, S157 (2004).

10. T. Alieva and M. Bastiaans, "Mode mapping in paraxial lossless optics," Opt. Lett. 30, 1461-1463 (2005).

11. M. A. Bandres and J. C. Gutiérrez-Vega, "Ince-Gaussian beams," Opt. Lett. 29, 144-146 (2004).

12. M. A. Bandres and J. Gutiérrez-Vega, "Ince-Gaussian series representation of the two-dimensional fractional Fourier transform," Opt. Lett. 30, 540-542 (2005).

13. J. C. Gutiérrez-Vega and M. A. Bandres, "Ince-Gaussian beams in a quadratic-index medium," J. Opt. Soc. Am. A 22, 306-309 (2005).

14. T. Alieva and A. M. Barbé, "Self-fractional Fourier images," J. Mod. Opt. 46, 83-99 (1999).

15. F. Gori, G. Guattari, and C. Padovani, "Bessel-Gauss beams," Opt. Commun. 64, 491-495 (1987).

16. E. Abramochkin and V. Volostnikov, "Spiral-type beams," Opt. Commun. 102, 336-350 (1993).

17. E. Abramochkin and V. Volostnikov, "Spiral-type beams: optical and quantum aspects," Opt. Commun. 125, 302-323 (1996).

18. E. G. Abramochkin and V. G. Volostnikov, "Spiral light beams," Phys. Uspekhi 47, 1177-1203 (2004).

19. Y. Y. Schechner, R. Piestun, and J. Shamir, "Wave propagation with rotating intensity distributions,” Phys. Rev. E 54, R50-R53 (1996).

20. R. Piestun and J. Shamir, "Generalized propagation-invariant wave fields,” J. Opt. Soc. Am. A 15, 3039-3044 (1998).

21. A. O'Neil and M. Padgett, "Axial and lateral trapping efficiency of Laguerre-Gaussian modes in inverted optical tweezers," Opt. Commun. 193, 45-50 (2001).

22. E. Abramochkin, S. Kotova, A. Korobtsov, N. Losevsky, A. Mayorova, M. Rakhmatulin, and V. Volostnikov, "Microobject manipulations using laser beams with nonzero orbital angular momentum," Laser Phys. 16, 842-848 (2006).

23. S. R. P. Pavani, A. Greengard, and R. Piestun, "Threedimensional localization with nanometer accuracy using a detector-limited double-helix point spread function system," Appl. Phys. Lett. 95, 021103 (2009).

24. A. N. B. Spektor and J. Shamir, "Singular beam microscopy," Appl. Opt. 47, A78-A87 (2008).

25. S. B. S. Fürhapter, A. Jesacher, and M. Ritsch-Marte, "Spiral interferometry," Opt. Lett. 30, 1953-1955 (2005).

26. P. Senthilkumaran, J. Masajadar, and S. Sato, "Interferometry with vortices," Int. J. Opt. 2012, 517591 (2012).

27. G. Gibson, J. Courtial, M. Padgett, M. Vasnetsov, V. Pas'ko, S. Barnett, and S. Franke-Arnold, "Free-space information transfer using light beams carrying orbital angular momentum," Opt. Express 12, 5448-5456 (2004).

28. G. Gbur and O. Korotkova, "Angular spectrum representation for the propagation of arbitrary coherent and partially coherent beams through atmospheric turbulence,” J. Opt. Soc. Am. A 24, 745-752 (2007).

29. G. Gbur and R. K. Tyson, "Vortex beam propagation through atmospheric turbulence and topological charge conservation," J. Opt. Soc. Am. A 25, 225-230 (2008).
30. F. Dubois, L. Joannes, and J.-C. Legros, "Improved threedimensional imaging with a digital holography microscope with a source of partial spatial coherence," Appl. Opt. 38, 7085-7094 (1999).

31. X. Ma and G. R. Arce, "PSM design for inverse lithography with partially coherent illumination," Opt. Express 16, 20126-20141 (2008)

32. J. Wang, J.-Y. Yang, I. M. Fazal, N. Ahmed, Y. Yan, H. Huang, Y. Ren, Y. Yue, S. Dolinar, M. Tur, and A. E. Willner, "Terabit freespace data transmission employing orbital angular momentum multiplexing," Nat. Photonics 6, 488-496 (2012).

33. J. C. Ricklin and F. M. Davidson, "Atmospheric turbulence effects on a partially coherent Gaussian beam: implications for free-space laser communication," J. Opt. Soc. Am. A 19, 1794-1802 (2002).

34. C. Schwartz and A. Dogariu, "Mode coupling approach to beam propagation in atmospheric turbulence," J. Opt. Soc. Am. A 23, 329-338 (2006)

35. Y. Yuan, Y. Cai, J. Qu, H. T. Eyyuboğlu, Y. Baykal, and O. Korotkova, " $M^{2}$-factor of coherent and partially coherent dark hollow beams propagating in turbulent atmosphere," Opt Express 17, 17344-17356 (2009)

36. M. Born and E. Wolf, Principles of Optics (Cambridge University, 2006).

37. P. De Santis, F. Gori, G. Guattari, and C. Palma, "Synthesis of partially coherent fields,” J. Opt. Soc. Am. A 3, 1258-1262 (1986).

38. Y. Qiu, H. Guo, and Z. Chen, "Paraxial propagation of partially coherent Hermite-Gauss beams," Opt. Commun. 245, 21-26 (2005)

39. F. Wang, Y. Cai, and O. Korotkova, "Partially coherent standard and elegant Laguerre-Gaussian beams of all orders," Opt. Express 17, 22366-22379 (2009).

40. F. Gori, M. Santarsiero, R. Borghi, and G. Guattari, "Intensitybased modal analysis of partially coherent beams with Hermite-Gaussian modes," Opt. Lett. 23, 989-991 (1998).

41. S. A. Ponomarenko, "A class of partially coherent beams carrying optical vortices,” J. Opt. Soc. Am. A 18, 150-156 (2001).

42. G. V. Bogatyryova, C. V. Fel'de, P. V. Polyanskii, S. A Ponomarenko, M. S. Soskin, and E. Wolf, "Partially coherent vortex beams with a separable phase," Opt. Lett. 28, 878-880 (2003)

43. L. Mandel and E. Wolf, Optical Coherence and Quantum Optics (Cambridge University, 1995).

44. J. A. Rodrigo, T. Alieva, and M. L. Calvo, "Programmable twodimensional optical fractional Fourier processor," Opt. Express 17, 4976-4983 (2009).

45. J. A. Rodrigo, T. Alieva, A. Cámara, O. Martínez-Matos, P Cheben, and M. L. Calvo, "Characterization of holographically generated beams via phase retrieval based on Wigner distribution projections,” Opt. Express 19, 6064-6077 (2011).

46. K. Sundar, N. Mukunda, and R. Simon, "Coherent-mode decomposition of general anisotropic Gaussian Schell-model beams," J. Opt. Soc. Am. A 12, 560-569 (1995).

47. R. Borghi, G. Guattari, L. de la Torre, F. Gori, and M. Santarsiero, "Evaluation of the spatial coherence of a light beam through transverse intensity measurements," J. Opt. Soc. Am. A 20 1763-1770 (2003).

48. T. Alieva and M. J. Bastiaans, "Self-affinity in phase space," J. Opt. Soc. Am. A 17, 756-761 (2000).

49. E. Baleine and A. Dogariu, "Variable coherence tomography," Opt. Lett. 29, 1233-1235 (2004).

50. F. O. Fahrbach, P. Simon, and A. Rohrbach, "Microscopy with self-reconstructing beams," Nat. Photonics 4, 780-785 (2010).

51. A. Cámara and T. Alieva, "Propagation of broken stable beams," J. Mod. Opt. 58, 743-747 (2011). 